\section{NCy 35}

DACARBAZINE INDUCED HUMAN LIVER DAMAGE, R.Paschke,M. Heine, J.Schmitz, E. Wetzel, J.M. Wdowinski, U. Schwedes

Phase I evaluation of Dacarbazine showed SGOT elevations in $50 \%$ and eosinophilia in $0,7 \%$. Thereafter hepatic veno occlusive disease(VOD) and budd chiari syndrome(BCS)have been reported as lethal complications of Dacarbazine therapy.Peripheral eosinophilia and eosinophilic infiltrates after repeated Dacarbazine therapy in some cases were interpreted as evidence of an allergic drug reaction. Since descriptions of an eosinophilia are missing in other reparts of VDO or BCS due to Dacarbazine therapy the pathophysiological mechanism of these lethal complications due to Dacarbazine remains to be elucidated. We describe a case of VOD due to Dacarbazine which showed a peripheral eosinophilia after the first cycle of Dacarbazine treatment along with signs of a toxic drug reaction. After the first therapy in 1980 lymph node enlargement recured in january 1984 in the right groin. Cytostatic treatment was startet with bleomycin $15 \mathrm{mg}$, vinblastine $10 \mathrm{mg}$, doxorubicin $40 \mathrm{mg}$ on february $23 \mathrm{rd}$ and Dacarbazine $400 \mathrm{mg}$ from february $23 \mathrm{rd}$ to february $27 \mathrm{th}$. On february $28 \mathrm{th}$ hepatic failure occured which was caused by VOD as demonstrated by post mortem examination. Two weeks after Dacarbazine therapy a previously not detected eosinophilia( $21 \%$, WBC 6 400) was observed.Because of the immediate rise of transaminases and bilirubin the ascites one day after chemotherapy and the histologic findings a toxic drug reaction due to DTIC must be assumed. Since it is known that a peripheral eosinophilia may be seen after tissue necrosis like in our case, a peripheral eosinophilia after Dacarbazine treatment is not evidence enough for an allergic drug reaction. Our observations suggest that peripheral eosinophilia so far interpreted as a sign of an allergic drug reaction due to Dacarbazine can be observed together obvious signs of a toxic mechanism of Dacarbazine induced VOD or BCS.Aetiology and significance of peripheral eosinophilia duxing Dacarbazine treatment await further clarification.

R.Paschke, II.medizinische Klinik, Klinikum Mannheim der Universität Heidelberg, Theodor-Kutzer-Ufer,680o Mannheim 1 .

\section{NCy 36}

COMBINATION OF AMINOGLUTETHIMIDE AND POLYCHEMOTHERAPY IN BREAST CANCER WITH BONE METASTASES K. Rieche, S. Dabag andL.Scharlau

The conventional chemo-hormonal therapy of breast cancer combines Tamoxifen plus chemotherapy. We conducted a non-randomised clinical study in patients (pats.) with predominant bone metastases of breast cancer by combining the in hibitor of aromatase Aminoglutethimide ( $A G$ )plus simultaneous polychemotherapy. Twenty-seven pats. entered the study so far. Twenty of the pats.had prior chemo-hormonal treatment. The mean age of the pats. was 59 years (range 37-75). Sixteen pats. received $A G$ plus polychemotherapy containing Adriamycin. This combination was preferred for thase pats. Who had prior treatment with CMF-regimen (Cyclophosphamide, Methotrexate, 5Fluorouracil)or for those who had additional visceral metastases or very progressive osteoiysis. Eleven pats. were given AG plus CMF. Twenty-six pats. of the study were"adequately treated, and 25 pats. were evaluable so far. Responses were evaluated 3 months after beginning of the treatment. The overall response rate was $56 \%$ ( $1 \mathrm{CR}, 13 \mathrm{PR}$ ). The median survival of all patients was 11 months. Nine of the 14 (64\%) evaluable pats. Who were given AG plus polychemotherapy containing Adriamycin achieved objective responses ( $1 \mathrm{CR}, 8 \mathrm{PR}$ ). In the group of 11 pats. Who received $A G$ plus CMF-regimen only 5 (45\%) responded (5PR). The difference in the response rates seem to be remarkable and need fur ther substantiation by continuation of the study.

Onkologische Abteilung am St. Josef-Hospital, Buerer Str. $47,0-465$ Gelsenkirchen

\section{Ifo 01}

EXPERIMENTAL BASIS OF MODERN IFOSFAMIDE THERAPY N. Brock and J. Poh 1

Ifosfamide (Ifo), like cyclophosphamide (Cp) belongs to the group of oxazaphosphorines. Regarding the profile of action of both compounds, there are important differences: Chemical structure: One of the two 2-chloroethyl groups of the exocyclic nitrogen mustard moiety of $\mathrm{CP}$ is transposed in Ifo onto the cyclic phosphamide nitrogen of the oxazaphosphorine ring.

Pharmacodynamics: Ifo differs from $C_{p}$ in the behaviour of the time constants for the curative and lethal action. Its cumulation of curative action is increased and that of the toxic action is reduced. Therefore fractionated dosage regimens or continuous infusion over 1 - 5 days increase the tolerability and remission rates with ifo.

Metabolism: Like Cp Ifo is also "activated" in the liver. As with $C p$, the basis of cytotoxic specificity and cancerotoxic selectivity is the oxidized oxazaphosphorine ring hydroxylated at the carbon 4 atom. "Side chain oxidation" with formation of chloracetaldehyde plays a more important role with Ifo than with $C p$.

Pharmacokinetics: The spontaneous toxification of activated Ifo is slower than that of $\mathrm{Cp}$, and the alkylation rate of the end product of Ifo toxification is much slower than that of nitrogen mustard phosphorid acid diamide which is the toxification product of $\mathrm{Cp}$.

Pharmacotherapeutic characteristics: 4-hydroxy-Ifo has the highest cytotoxic specificity of all oxazaphosphorine metabolites. Therapeutic comparisons conducted with optimal dosages confirm the superior therapeutic range of Ifo in numerous experimental animal tumours.

Detoxification: The wider therapeutic range of Ifo is based on the reduction in toxicity by dose fractionation. A further increase in selectivity was made possible by an effective uroprotector (mesna) which obviates the therapylimiting urotoxic side effects, esp. hemarrhagic cystitis. Asta-Werke AG Degussa Pharma Gruppe, Postfach 140129 D-4800 Bielefeld 14

\section{Ifo 02}

IFOSFAMIDE (IFO) - DOSING AND SCHEDULING

W. Brade*, H.O. Klein, K. Herdrich*

In the early development of IFO no significant toxicity was found after single doses of up to $2.9 \mathrm{~g} / \mathrm{m}^{2}$ while CNS symptoms and urotoxicity became dose limiting above $5 \mathrm{~g} / \mathrm{m}^{2}$ (Cohen et al., Canc. Chemoth. Rep. 59, 751 (1975)). Because of experimentally shown higher cumulative efficacy and lower toxicity (Brock, Verhdl. Dtsch. Krebsges., Fischer-Verlag 1978), dose fractionation was common in recent years, i.e. single doses between 1.6 and $2.5 \mathrm{~g} / \mathrm{m}^{2}$ given over 3-5 days. By giving the uroprotector Mesna the former dose limiting urotoxicity can be controlled and IFO doses have been escalated either up to $5 \mathrm{~g} / \mathrm{m}^{2}$ in a $30 \mathrm{~min}$. intravenous continuous infusion (i.v.-c.i.) or to maximum tolerated doses of $3.4 \mathrm{~g} / \mathrm{m}^{2}$ given as $i . v .-c . i$. over $5 \mathrm{con}-$ secutive days (Klein et al., Cancer 54, 1198 (1984)). Repetttive dosing over $4-5$ consecutive days led to increased serumconcentrations of 4-hydroxy-IFO (Klein et al. Cancer 54, 1193 (1984)) and to a shortened beta-phase serum half life of IFO (Piazza et al., Cancer 54, 1187 (1984)), which suggests increased metabolic activation. Due to the prolonged urinary elimination of IFO metabolites and the short half life of Mesna in urine adequate adaptation of Mesna dose to newly developed IFO schedules is required. In combination chemotherapy presently applied IFO doses range from $1.2 \mathrm{~g} / \mathrm{m}^{2} / \mathrm{d}_{1-5}$ over $3 \mathrm{~g} / \mathrm{m}^{2} / \mathrm{d}_{1+2}$ to $5 \mathrm{~g} / \mathrm{m}^{2}$ in $24 \mathrm{hr}$ i.v. $-\mathrm{c}$. i.

Since the application of higher IFO doses under uroprotection by mesna may increase efficacy but might also produce secondary toxicities as CNS symptoms, myelosuppression and nephrotoxicity, additional clinical trials are needed, which allow direct comparison on efficacy and tolerance of different IFo plus Mesna schedules.

* Asta-Werke AG, Degussa Pharma Gruppe, Medizinische Abteilung, Daimlerstraß 25, D-6000 Frankfurt 1

Medizin. Univ.-Klinik Köln, Joseph-Stelzmann-Str. 9 D -5000 Köln 41 